\title{
The striatum multiplexes contextual and kinematic information to constrain motor habits execution
}

\author{
Pavel E Rueda-Orozco ${ }^{1-4}$ \& David Robbe ${ }^{1-4}$ \\ The striatum is required for the acquisition of procedural memories, but its contribution to motor control once learning has \\ occurred is unclear. We created a task in which rats learned a difficult motor sequence characterized by fine-tuned changes in \\ running speed adjusted to spatial and temporal constraints. After training and extensive practice, we found that the behavior \\ was habitual, yet tetrode recordings in the dorsolateral striatum (DLS) revealed continuous integrative representations of running \\ speed, position and time. These representations were weak in naive rats that were hand-guided to perform the same sequence \\ and developed slowly after learning. Finally, DLS inactivation in well-trained animals preserved the structure of the sequence \\ while increasing its trial-by-trial variability. We conclude that, after learning, the DLS continuously integrates task-relevant \\ information to constrain the execution of motor habits. Our results provide a straightforward mechanism by which the basal \\ ganglia may contribute to habit formation and motor control.
}

In many of our everyday actions, we perform specific sequences of movements with kinematic parameters (for example, movement time, speed, trajectory) precisely adjusted to task-specific constraints ${ }^{1}$. Examples can be seen in the actions involved in driving a car, playing sports, using tools and performing arts. Several weeks to several months of training are necessary for the acquisition of these behaviors, which are generally referred to as procedural memories or motor skills ${ }^{2}$. With practice, these skills can be performed automatically, and there is converging evidence from lesion and physiological studies using motor sequence tasks in rodents ${ }^{3-10}$, monkeys ${ }^{11-14}$ and humans ${ }^{15,16}$ that the sensorimotor region of the striatum (the DLS in rodents) contributes to the execution of well-learned procedural memories, especially motor habits ${ }^{17}$. Nevertheless, the exact nature of this contribution remains unclear ${ }^{18}$ and debated ${ }^{19}$, possibly because action execution is the readout of higher order processes such as motor planning or action selection ${ }^{18}$. The clarification of this ambiguity is difficult for two reasons. First, behavioral performance during motor sequence tasks is generally quantified through global metrics (for example, percentage of correct trials, average reaction time, number of lever presses), which overlook changes in kinematic parameters of movements occurring during learning (for example, movements speed and trajectory, increased stereotypy of behavior and sensory stimuli). Second, as a result of the massive sensorimotor input of the striatum ${ }^{20}$ and its indirect connection toward motor circuits ${ }^{21}$, striatal spiking activity can be influenced by or influence kinematic parameters of action execution. Thus, during and after motor learning, it has not been established whether striatal spiking activity primarily reflects the acquisition of highlevel motor control function (for example, action planning, storage of motor programs), changes in low-level parameters (sensory and proprioceptive stimuli, dynamics of movements) that occurred during learning ${ }^{12}$ or specifically contributes to precise kinematic parameters associated with accurate execution ${ }^{12,18,19,22}$. Addressing this problem requires a task that dissociates low-level from highlevel processes associated with motor learning.

\section{RESULTS}

We designed a task for rats that favors the generation of a motor sequence with fine-tuned kinematic parameters that are easily quantifiable. Specifically, we customized a motorized treadmill and trained rats to obtain rewards according to a spatiotemporal rule. Once the treadmill was turned on, animals could stop it and receive a drop of sucrose solution by entering a 'stop area' located at the front of the treadmill (Fig. 1a). In addition to this spatial rule, a temporal constraint was added: stopping of the treadmill was only effective if animals waited at least $7 \mathrm{~s}$ (goal time) before entering the stop area (correct trials; Fig. 1b). If animals entered the stop area before the goal time, an error sound was played and they were forced to run for 20 s (incorrect non-rewarded trials; Fig. 1b). Initially, rats accelerated forward as soon as the treadmill was turned on and entered the stop area before the goal time, resulting in a majority of incorrect trials (Fig. 1c and Supplementary Video 1). After extensive training, rats executed a stereotyped sequence that could be divided in three overlapping phases: passive displacement from the front to the rear portion of the treadmill, stable running, and acceleration across the treadmill to enter the stop area (Fig. 1c, Supplementary Fig. 1 and Supplementary Video 2). The high level of stereotypy revealed by the trajectories of the animal was also demonstrated when tracking the position of the left forelimb (Supplementary Fig. 2). Learning occurred in a two-step process. Within a few sessions, animals learned to perform the 'front-rear-front' motor sequence, but they entered the stop area just before the goal time in the majority of the trials (sessions 15-45; Fig. 1d), which resulted in a low percentage of correct trials (Fig. 1e). Then, during the longest part of the training, animals

${ }^{1}$ INSERM, U901, Marseille, France. ${ }^{2}$ Aix-Marseille University, UMR 901, Marseille, France. ${ }^{3}$ INMED, Marseille, France. ${ }^{4}$ Department of Systems Neuroscience, Institut d'Investigacions Biomèdiques August Pi i Sunyer (IDIBAPS), Barcelona, Spain. Correspondence should be addressed to D.R. (david.robbe@inserm.fr).

Received 30 July 2014; accepted 16 December 2014; published online 26 January 2015; doi:10.1038/nn.3924 
Figure 1 A fixed interval estimation task adapted to treadmill locomotion favored the learning of a stereotyped running sequence. (a) Behavioral apparatus. (b) Task description. (c) Running trajectories (left) and entrance times in the stop area (right) for two sessions, before (top) and after (bottom) learning. $(\mathbf{d}, \mathrm{e})$ Entrance times in the stop area (median \pm first and third quartiles; d) and percentage of correct trials (e) for all the training sessions of a single animal.

progressively adjusted the kinematic parameters of execution to delay their entering in the stop area and increase their proficiency (sessions 30-100; Fig. 1d,e).

We next examined whether this learning process leads to goal-directed or habitual performance of the running sequence. Motor habits are difficult to break and a classical test to probe the strength of habits is contingency degradation ${ }^{23,24}$. We designed two tests of contingency degradation that were adapted to our task. First, we shortened the treadmill (Fig. 2a) after extensive training ( $>80$ daily sessions), when the animals had reached stable performance ( $\geq 3$ consecutive sessions with $\geq 72.5 \%$ of correct trials). We observed that, during several sessions following treadmill shortening, the animals persisted in performing the task using the previously learned kinematic parameters, yielding a majority of premature entrances into the stop area (Fig. 2a). Notably, although the motor sequence was qualitatively identical before and after shortening (Fig. 2b), a great number of sessions were necessary for the animals to learn the new kinematic parameters adapted to the shorter treadmill (Fig. 2c). Second, we trained a separate group of animals to enter the stop area after a short goal time (4 or $5 \mathrm{~s}$ ) and, once the animals reached stable performance, the goal time was increased to $7 \mathrm{~s}$. After this change, animals persisted in entering in the stop area just after the original goal time for several sessions (sessions 116-118; Fig. 2d) and it took the animals several sessions to return to the learning criterion $(37,16$ and 10 sessions for rats 7,9 and 10 , respectively). In both tests, behavioral persistence occurred despite the fact that rats occasionally performed correct trials under the new conditions (Fig. 2a,d). In some cases, several sessions after the change, the animals relapsed and performed the motor sequence using the previously learned kinematic parameters (Fig. 2c,d). This is suggestive of a motor habit that is difficult to break.

Finally, we examined the effect of reward devaluation on task performance 23,24 . This was achieved by giving the animals free access to the sucrose solution before a probe session. We performed this procedure during training (Fig. 2e) and found that, when animals had not yet reached behavioral proficiency, they disengaged from the task during the devaluation test (Fig. 2e). However, when we repeated the same procedure after further training, animals were less and less sensitive to reward devaluation (Fig. 2e). Altogether, the results obtained in this set of behavioral manipulations are indicative of an habitual performance of the motor sequence after learning and extensive practice.

Next, we performed tetrode recordings in the DLS of rats with stable proficient performance in the task and examined whether neuronal correlates relevant for execution of the running sequence could be extracted from the spiking activity of well-isolated units ( $n=391$ from 3 rats; Supplementary Fig. 3). Consistent with previous works, a minority of task-modulated units responded to the warning cue preceding the start of the treadmill (Supplementary Fig. 4) ${ }^{25}$
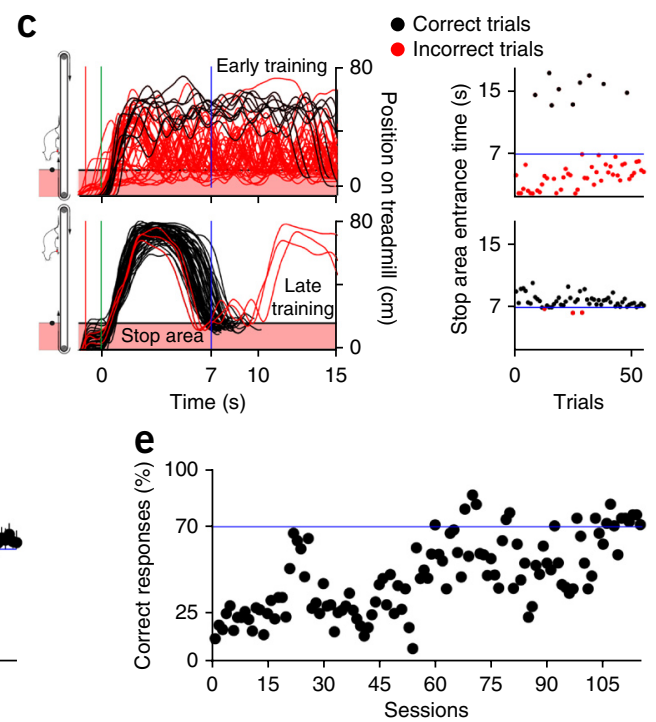

or to reward delivery (Supplementary Fig. 5) ${ }^{26}$ or fired in bursts synchronized with limb movements (Supplementary Fig. 6) 27,28 . Still, as a population, striatal neurons displayed continuous sequential modulation of their spiking activity during task performance (Fig. 3a,b). To characterize the relationship between spiking activity and execution of the motor sequence, we correlated instantaneous firing rates with the main behavioral variables (position on the treadmill, running speed and acceleration) and time. We found that a large fraction of these neurons displayed notable linear correlations between firing rate and either running speed (Fig. $\mathbf{4 a - c}$ ) or position of the animal on the treadmill (Fig. 4d). The specificity of these correlations was quantified by computing Pearson's partial correlation coefficients (Fig. 4a-d) and was confirmed when plotting error trials (Supplementary Fig. 7). Correlations between firing rate and running speed could not be accounted by transitions between trotting and galloping (Supplementary Fig. 8). In addition, some units had their firing rate co-modulated by time, position and speed (Fig. 4e,f). Finally, striatal spiking activity was weakly correlated with acceleration (Fig. $\mathbf{4 g}$ ). Next, we used a multiple regression analysis to quantify the extent to which the firing rates of striatal units could be explained by a linear combination of the main behavioral variables (time, position, speed and acceleration; Online Methods). First, the results obtained using partial correlation analysis were confirmed by examining the $F$ values of each coefficient (medians of the $F$ values for speed, position, time and acceleration were 877, 342, 151 and 70, respectively). Second, at the population level, the multiple correlation coefficients were consistently larger than the partial correlation coefficients (Fig. 4g), suggesting that firing rates were generally influenced by more than one variable (Fig. $\mathbf{4 b}, \mathbf{e}$ ). This possibility was confirmed by comparing regression models that took into account a single predictor with models that took into account several (2,3 or 4) predictors (Supplementary Fig. 9). Finally, the proportion of units modulated by position, speed and acceleration and the predominance of linear correlations were confirmed with a distinct statistical method that did not rely on linear or monotonic relationships between spiking activity and behavioral variables (Supplementary Fig. 10). Altogether, these results indicate that, during habitual execution of the running sequence, spiking activity in the DLS is principally correlated with running speed and the position of the animal on the treadmill. In addition, our data also suggest that neurons of the DLS multiplex information from several task-related variables. 
Figure 2 Evidence for habitual performance of the running sequence. (a) Entrance times (median \pm first and third quartiles) and percentage of correct trials, before and after shortening the treadmill. (b) Normalized averaged trajectories during the last session before treadmill shortening (orange trace) and the next 40 sessions (gray, all sessions; blue and red, sessions 5 and 40 after shortening). (c) Trajectory changes following treadmill shortening. Colors are presented as in $\mathbf{b}$. Rectangle in $\mathbf{b}$ shows the time at which the trajectory changes were computed. (d) Stop area entrance times (left, trial by trial; right, median \pm first and third quartiles) before and after changing the goal time. (e) Stop area entrance times (left, trial by trial; right, median \pm first and third quartiles) before and during reward-devaluation sessions (blue) performed at different times during training. Red dashed lines indicate goal times $(\mathbf{d}, \mathbf{e})$. All the data are taken from single animals and were reproduced in at least three animals.

The robust linear relationship between striatal activity and running speed could be a correlate of running speed control. If this was the case, this relationship should improve when correlating running speed at a given time with preceding spiking activity and decrease when correlating running speed at a given time with subsequent spiking activity. Such a temporal relationship between a 'leading' neuronal activity and a 'following' behavior has been observed in the parietal cortex $^{29}$ and was revealed by systematically shifting the spike times of the recorded units either forward or backward in time and recomputing correlation coefficients for different time shifts. In contrast with what was observed in the parietal cortex ${ }^{29}$, we found that, at the population level, the correlation coefficients between firing rate and running speed were equally affected when spike times were shifted forward or backward relative to behavior (Fig. 5a-c). The analysis revealed that high correlation values were maintained for time shifts ranging from -0.25 to $+0.25 \mathrm{~s}$ (Fig. 5b,c). This result was confirmed by randomly jittering the spikes times of each unit on a trial-by-trial basis over different timescales and recomputing the correlation coefficients (Fig. 5d). We compared the decay of the correlation coefficients for different jitters with the auto-correlation function of the running
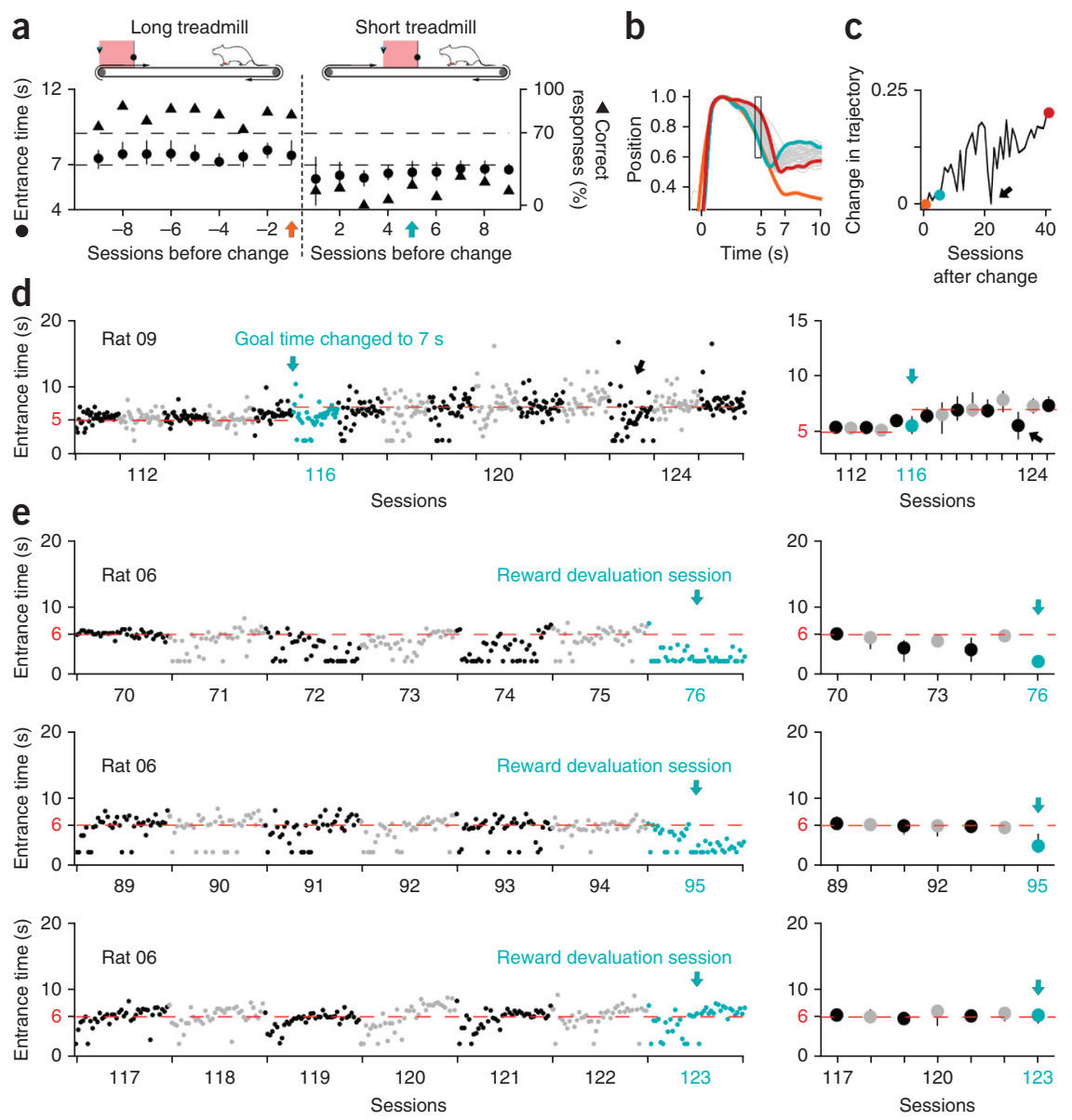

speed profiles. We found that changes in running speed occurred slightly faster than changes in neural activity (Fig. 5d). This result is more compatible with a continuous modulatory function of the speed-related spiking activity than a sharp instruction signal generating the running sequence on a moment-to-moment basis.

Neuronal activity in the region of the DLS in which we performed electrophysiological recordings is known to be sensitive to sensory stimulation of the limbs, trunk, neck, head and whiskers ${ }^{30}$. To rule out the possibility that position and speed-related modulations of firing rate passively reflect low-level sensorimotor activity that became stereotyped during learning ${ }^{12}$, we performed recordings in the DLS of naive rats $(n=3)$ hand-guided by the experimenter to perform the running sequence. For this purpose, when the treadmill started,
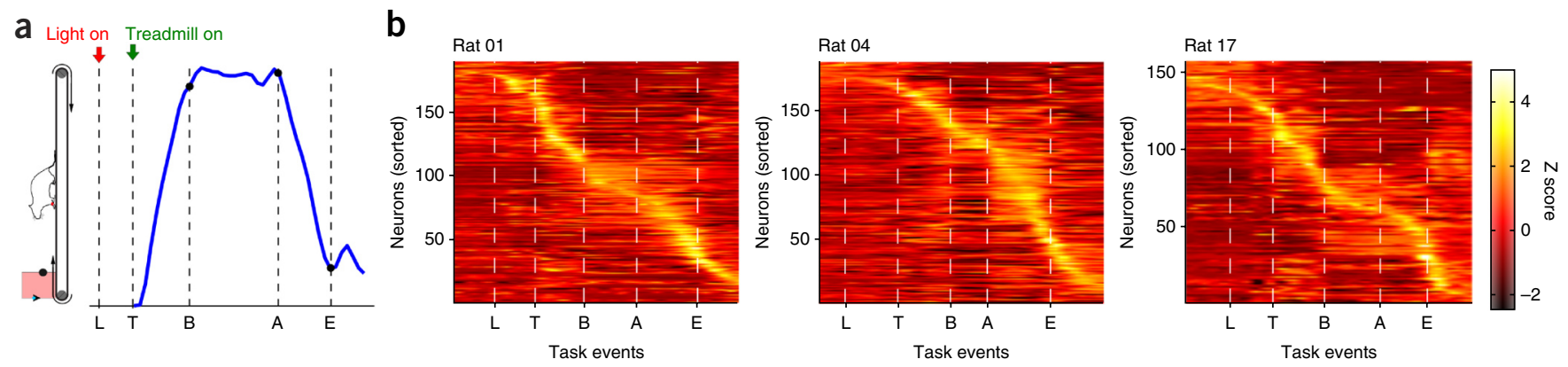

Figure 3 Continuous sequential modulation of striatal spiking activity during sequence execution. (a) Animal's trajectory (blue) during a single trial and task events: L, light on; T, treadmill on; B, animal reaches the back of the treadmill; A, animal accelerates to cross the treadmill; E, animal reaches the stop area or terminates the sequence. (b) Averaged modulations of the firing rate of all recorded units expressed as $Z$ score and sorted in time, during execution of the sequence, after learning and extensive practice. 
Figure 4 Linear and integrative representation of running speed, position and time after learning. (a-e) Five illustrative units with firing rate correlated with running speed $(\mathbf{a}-\mathbf{c})$, position on the treadmill (d), or a combination of time, position and speed (e). Left, spike rasters (top) and average peri-event histograms (bottom), aligned to the end of the motor sequence (a-d) or treadmill start (e). Middle scatter plots show firing rate versus best correlated task variable (mean \pm s.d.). Right, partial and multiple correlation coefficients between firing rate and task variables ( ${ }^{*} P<0.001$, Pearson's partial correlation coefficient). (f) Two illustrative trials taken from the unit shown in e, with trajectories (top) and instantaneous firing rates (bottom).

(g) Partial and multiple correlation coefficients (absolute values) between firing rate and task variables, sorted for all the recorded units (three rats, same color code than a-e). Correlation coefficients with $P<0.001$ are on the right side of the dashed line.

the experimenter used a rectangular plate to gently push the animal toward the rear of the treadmill and to maintain its position for a few seconds. The plate was then removed and the rats accelerated to cross the treadmill (Fig. 6a). To keep learning minimal, we did not give hand-guided animals rewards during these sessions. Running trajectories were highly similar in the well-trained and handguided animals (Fig. 6b). To our surprise, the sequential modulation of striatal units observed in well-trained animals (Fig. $3 \mathbf{b}$ ) was preserved in the hand-guided naive animals (Supplementary Fig. 11). Next, we examined possible differences in neuronal representation of the behavioral variables between well-trained and hand-guided animals. Partial and multiple correlation analyses revealed that the correlation coefficients between firing rate and both running speed and position were strongly reduced in the hand-guided animals compared with trained animals (well trained versus hand guided, $P<0.001$; Fig. $\mathbf{6 c}, \mathbf{d})$. The correlation coefficients between firing rates and time and acceleration were less affected (well trained versus hand guided: acceleration, $P=0.006$; time, $P=0.044$; Fig. $6 \mathbf{c}, \mathbf{d}$ ), probably because these coefficients were already low in well-trained animals (Fig. 4g). The decrease in linear representation of speed and position was strong and could not be explained by differences in running speed between hand-guided and well-trained animals (Supplementary Fig. 12). One possibility is that position- and speed-related activities are dampened as a result of impaired vision by the guiding plate. At the population level, the similar timing and amplitude of striatal firing rate modulations in hand-guided and well-trained animals argue against such a possibility (Fig. 3b and Supplementary Fig. 11). To directly address this issue, we took advantage of the fact that hand-guided animals sampled the same range of positions on the treadmill with the plate (when guided from front to rear) and without it (when running freely from rear to
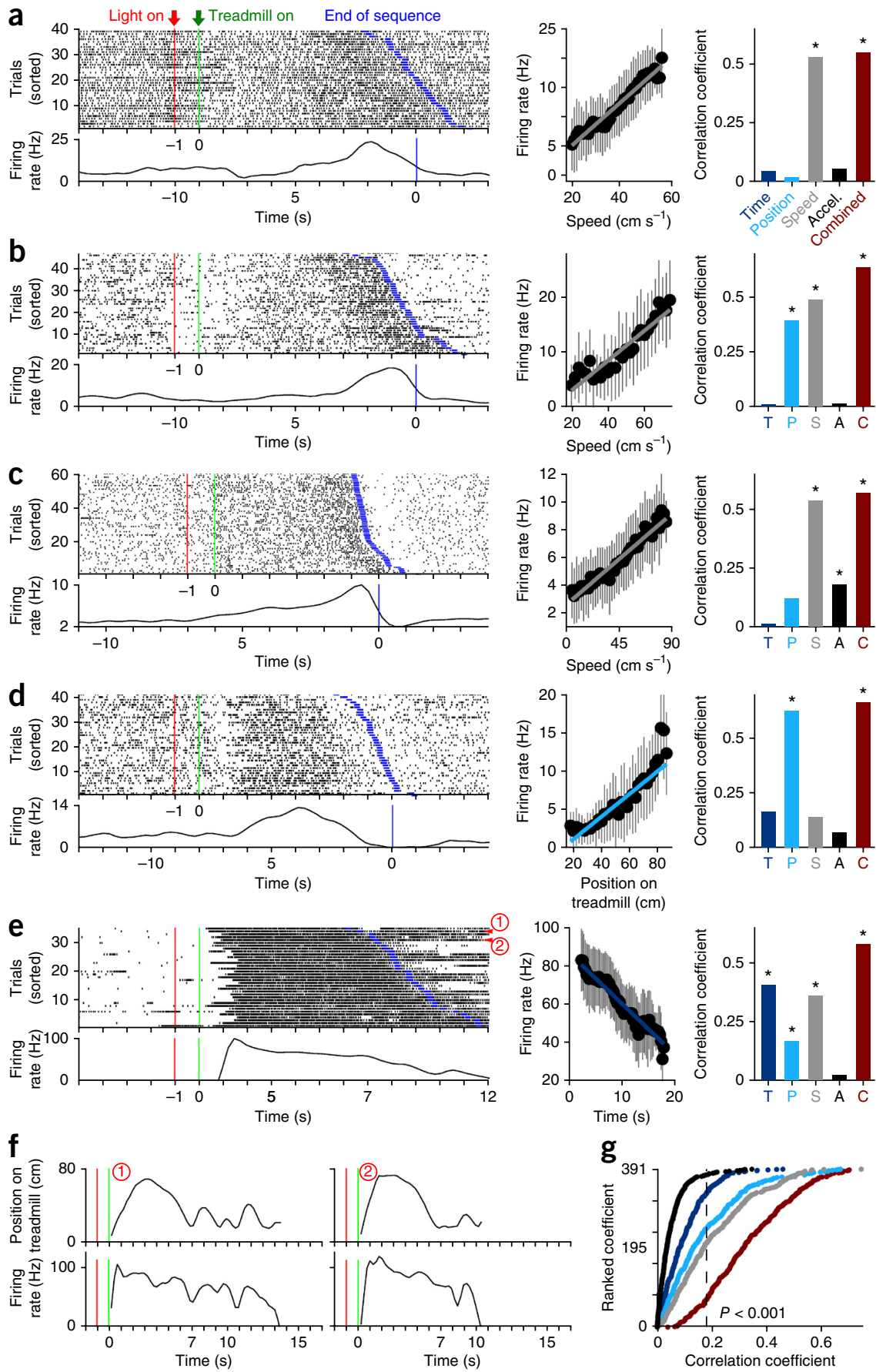

front). Partial correlation coefficients between firing rate and position, were identical in both conditions (median \pm s.d.; $r=0.097 \pm$ 0.1 , no plate; $r=0.089 \pm 0.07$, plate; $P=0.31$ ). Taken together, these results demonstrate that position and speed representations are not byproducts of the stereotyped sensorimotor activity.

This conclusion was reinforced by the study of one rat for which, following 1 week of hand-guided sessions, we continued to record neuronal and behavioral activity during 60 training sessions (that is, no more guiding plate; sucrose delivery for correct trials and long penalty runs for incorrect trials; Fig. 6e). The animal's behavioral performance was poor during the initial training sessions, but improved progressively and the front-rear-front motor sequence was learned (Fig. 6e,f). The strength of task representation in the DLS at 
Figure 5 Speed-related spiking activity changes more slowly than running speed. (a) Spike times were systematically shifted forward (yellow) or backward (blue) relative to behavior. (b) Firing rate versus speed (mean \pm s.d.) for forward and backward shifts, for the three illustrative speed-correlated units shown in Figure 4. (c) Partial correlation coefficients between firing rate and running speed, recalculated for each time shift (median, all recorded units). (d) Partial correlation coefficients between firing rate and running speed computed after randomly jittering the spike times (black) over increasing timescales. Normalized auto-correlation function of instantaneous running speeds (gray; median, all sessions). * and horizontal lines, $P<0.05$ versus central values (vertical dash lines), Kruskal-Wallis and Tukey's HSD test.

different times during training was quantified using the median of the multiple correlation coefficients distributions between firing rates and time, position, speed and acceleration. The strength of task representation was similarly low during the stereotyped hand-guided and early training sessions (Fig. 6f), confirming that the guiding plate had a minor effect on position and speed representations. Noticeably, task representation increased $\sim 20$ sessions after the behavioral performance became stereotyped and dominated by rewarded trials (Fig. 6f). Taken together, these results demonstrate that representations in the DLS are not mere byproducts of the increased behavioral stereotypy associated with motor learning, but emerge after extensive repetition of the correct motor sequence.

Our electrophysiological results revealed that, after learning and extensive practice of a motor sequence, the DLS displayed positionand speed-related signals. The fact that the speed-related signal evolves more slowly than the running speed itself suggests that the DLS may modulate or guide the execution of the motor sequence, but does not generate the whole sequence on a moment-by-moment basis. To test this hypothesis, we transiently perturbed neuronal activity in the DLS of highly trained animals ( $n=7$ from 4 rats) through bilateral injections of muscimol at a dosage that elicited minimal effects

a
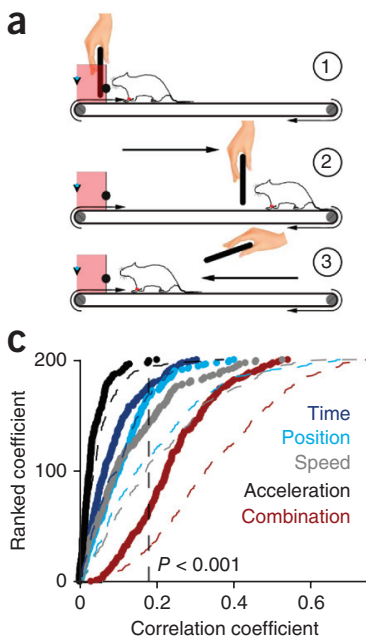

e

Hand-guided

sessions Training sessions
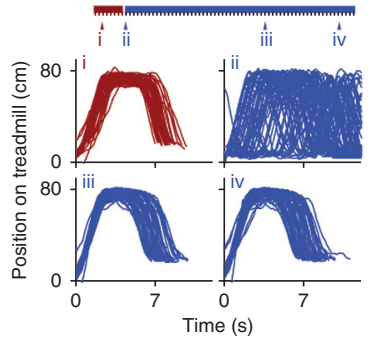

b

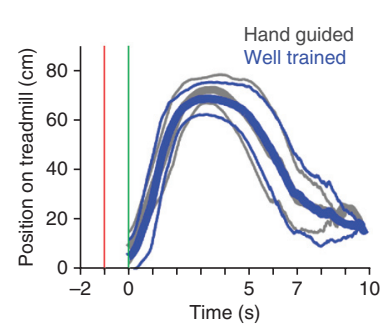

d

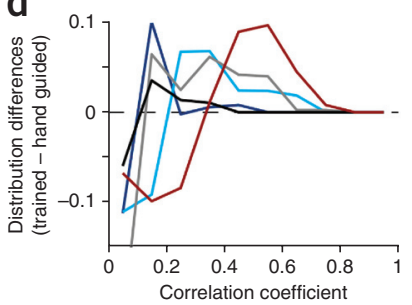

f

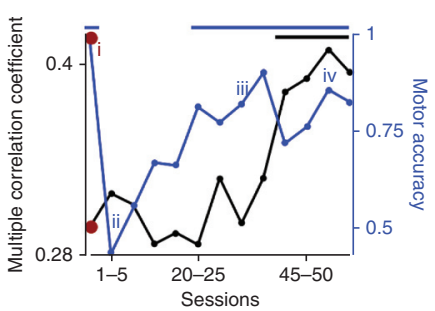

a

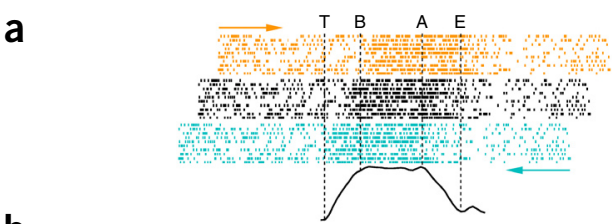

b
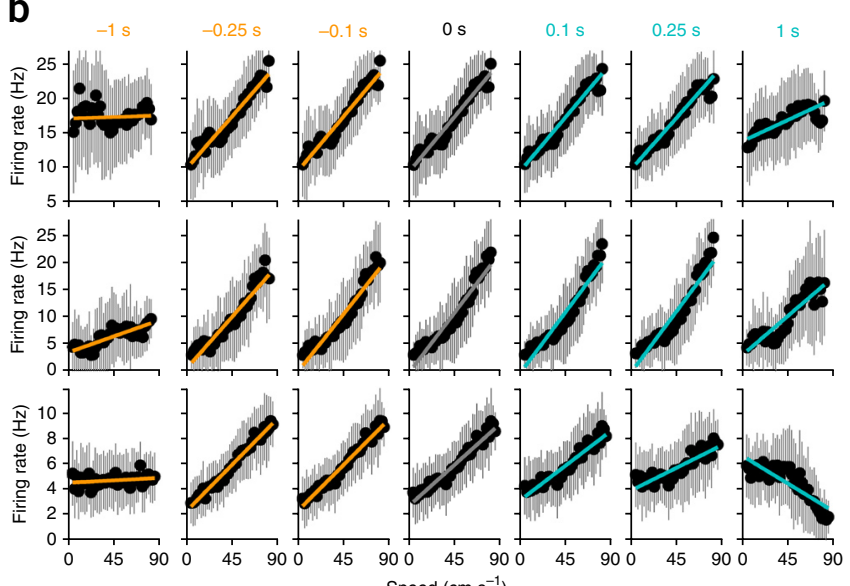

C
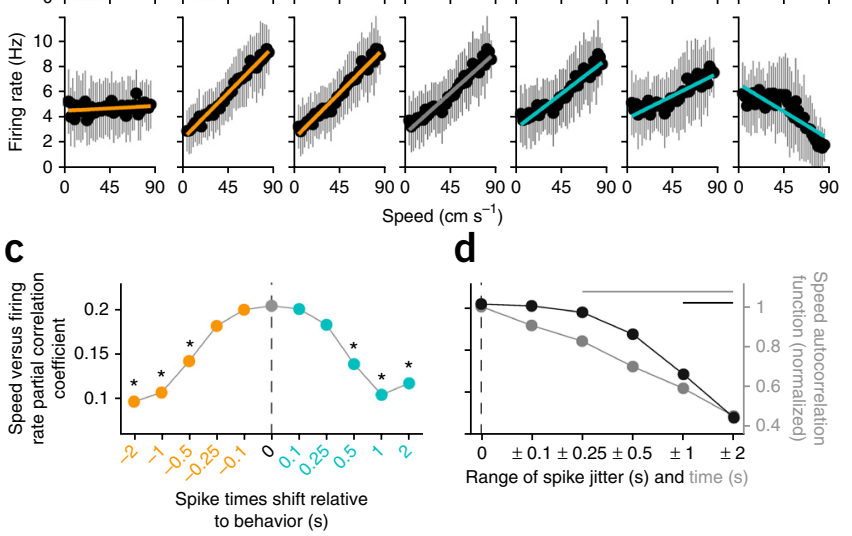

d

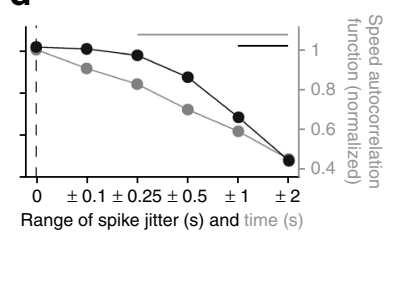

on locomotion (50 ng $\mu \mathrm{l}^{-1}, 1 \mu \mathrm{l}$ per site ${ }^{31}$; Supplementary Figs. 13 and 14). The variability of the entrance times in the stop area was markedly and reversibly increased following muscimol injections (Fig. 7a,b), yet there was no systematic change in the mean trajectory across experiments: the overall structure of the running sequence was always preserved (Fig. 7c and Supplementary Fig. 15). This result, and the fact that rats in the muscimol condition reached fast running speeds (Supplementary Fig. 14c,d), discount the possibility that a decrease in running speed is the cause of the increased variability in entrance times. The similar mean trajectories and number of rewards received (Supplementary Fig. 15a) before and after muscimol injections suggest that animals were similarly engaged in the task in both conditions. Another possibility is that, following muscimol injections, animals were confused and did not properly start the task. This was not supported by the fact that average behavior was preserved following muscimol injections (Supplementary Fig. 15d-f). Moreover,

Figure 6 Position and running speed representations in the DLS are specific of motor habits. (a) Hand-guided control task. (b) Trajectories (mean \pm s.d., single sessions) of a hand-guided (gray) and a well-trained (blue) animal. (c) Partial and multiple correlation coefficients between firing rate and task variables (absolute values) sorted for all the units, in the control task (rats 5, 15 and 17, solid dots). For comparison, dashed lines indicate scaled distributions of correlation coefficients computed from well-trained animals. (d) Difference in the cumulative distributions of the correlation coefficients in well-trained and hand-guided animals. (e) Recording schedule for rat 17 (top) and running trajectories during a hand-guided session (i), the first task training session (ii), once the sequence is learned (iii) and during overtraining (iv). (f) Motor accuracy (blue) and task representation in the DLS (black, median of the multiple correlation coefficients) of rat 17 during all of the hand-guided (red) and training sessions. Horizontal lines, $P<0.05$ versus first data point during training, Kruskal-Wallis and Tukey's HSD test. 
Figure 7 Inactivation of the DLS increases trial-by-trial variability of running sequence execution. (a) Stop area entrance times (top) and their variance (bottom) for the last 40 trials of single behavioral sessions $1 \mathrm{~d}$ before injection (black), 10 min after bilateral injections of muscimol (blue, $50 \mathrm{ng}$ in $1 \mu \mathrm{l}$ ) or saline (green, $1 \mu \mathrm{l}$ ) and $1 \mathrm{~d}$ after injection (gray). (b) Group effect for muscimol and saline injections.

(c) Average trajectories in a single experiment (same color code as in a). (d) Running speeds aligned relative to stop area entrance times for one session before (left) and 10 min after (right) muscimol injections. Dashed red lines indicate treadmill speed. (e) Group effect for muscimol injections. $(\mathbf{f}, \mathbf{g})$ Average and variance of the time spent by the animals in the rear portion of the treadmill (holding time). (h,i) Adjustment index (median \pm first and third quartiles) in control and muscimol conditions, after error trials (h, entrance time < goal time) and after trials in which animals entered the stop area at least $3 \mathrm{~s}$ after goal time (i). ns indicates not significant, ${ }^{*} P<0.05$, Kruskal-Wallis and Tukey's HSD test $(\mathbf{b}, \mathbf{e}-\mathbf{g})$ and Wilcoxon rank-sum test $(\mathbf{h}, \mathbf{i})$.

when we focused on trials in which animals correctly started the sequence, the increased variability was still apparent after muscimol injection (Supplementary Fig. 15b,c).

To determine the source of increased variability in entrance times after muscimol injections, we aligned speed time courses relative to entrance times and plotted them over the last $2.5 \mathrm{~s}$ preceding the termination of the sequence (Fig. $7 \mathbf{d}$ ). In the control condition, the speed time courses were highly stereotyped and consisted of an increase in running speed followed by a sharp deceleration (Fig. 7d). After muscimol injection, the speed time courses became highly variable, and this effect was consistently observed across experiments (Fig. 7d,e). Examination of the speed time courses during intra-DLS muscimol injection (Fig. 7d) suggested that an alteration in the timing at which rats initiated the final acceleration to cross the treadmill could not, by itself, explain the increased variance in arrival times. To further confirm this, we quantified the time the animals spent in the back of the treadmill and found no systematic change across experiments between control and muscimol conditions (Fig. 7f,g). These behavioral analyses show that both the timing and magnitude of the speed changes are altered after DLS perturbation.

An expected correlate of habitual performance is that, after an occasional error, proficient animals immediately adjust their performance on the next trial. We defined an adjustment index as the difference in entrance time between the error trial and the next trial divided by the size of the error. As expected, in the control condition, the indexes were mainly above 1 , but, after muscimol injection, the indexes dropped (Fig. 7h). Trials in which animals entered in the stop area several seconds after the goal time were not considered as errors, but were associated with an increased effort. Well-trained animals rarely performed twice in a row such 'long' trials and, in the control condition, the adjustment indexes after long trials (entrance time $\geq$ goal time $+3 \mathrm{~s}$ ) were in average slightly inferior to $1 \mathrm{~s}$. This immediate behavioral adjustment was impaired after muscimol injections (Fig. 7i). Altogether, after perturbation of DLS neuronal activity with b

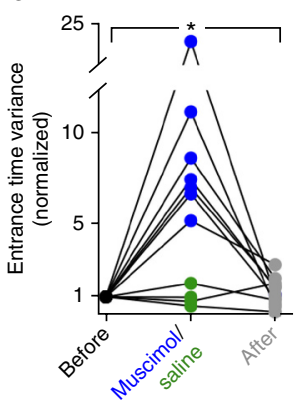

C
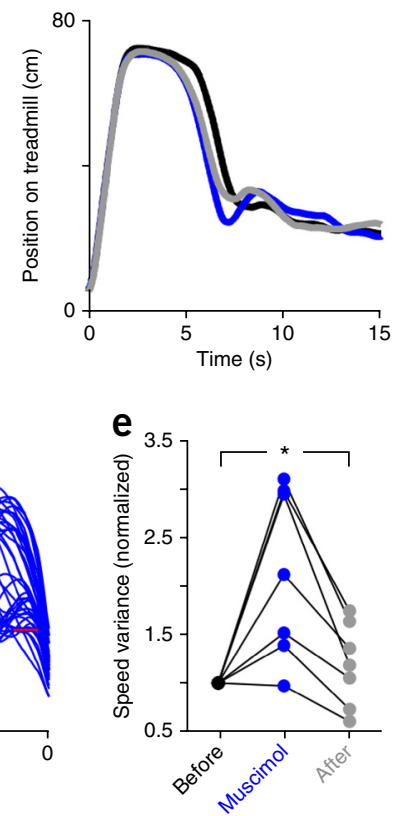

i
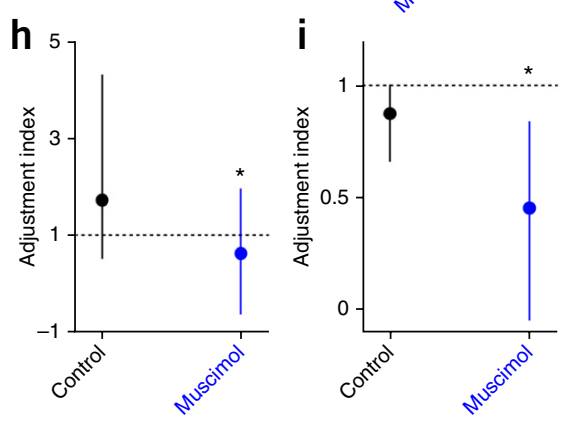

muscimol, the animals' capacity to perform the running sequence was spared, but execution became highly variable. This increased variability was associated with a difficulty of the animals to run at the right speed at the right time and impaired adjustment of performance after incorrect trials.

\section{DISCUSSION}

There are two confounding factors when studying the function of the DLS during motor learning. First, striatal spiking activity can both modulate actions ${ }^{32,33}$ and be modulated by actions ${ }^{27,28}$ through indirect projections toward motor circuits ${ }^{21}$ and a wide range of excitatory sensorimotor input ${ }^{20}$, respectively. Second, during learning, the dynamics of movements and associated sensory stimuli will change and eventually become stereotyped ${ }^{2}$. Thus, the inference of striatal mechanisms on the basis of changes in spiking dynamics during motor learning is non-trivial and has been subject to debate ${ }^{12,18,19}$. We found that, during habitual execution of a motor sequence, DLS neurons continuously represent in a combinatorial and linear manner contextual (time and position of the animal on the treadmill) and motor (running speed) information relevant for accurate performance. Importantly the representation of these variables was weak in naive rats performing the same motor sequence under the guidance of the experimenter and developed after learning. Thus, these representations do not merely reflect the stereotyped dynamics of "lowlevel" task parameters but are a signature of motor habits. Notably, we found that perturbing these signals spared the average running trajectories, but increased trial-by-trial variability and impaired the 
animals' capacity to adjust their performance after incorrect trials. Altogether the integrative neuronal representations of speed, position and time that we discovered in the DLS, combined with the type of behavioral impairment observed after its neuronal inactivation, suggest that an important function of this region is to continuously constrain the execution of motor habits.

To control the trajectories of the naive animals during hand-guided sessions, we used a guiding plate that could have altered the animals' vision and artificially weakened position and speed representations. First, this possibility is unlikely because the visual cortex targets the dorsomedial striatum, not the DLS ${ }^{34}$. Second, in the hand-guided recording sessions, we found no difference in position representation when we separately analyzed the portion of the trials in which the plate was used (animals guided from the front to the rear portion of the treadmill) and the portion of the trials in which the plate was removed (when animals accelerated to cross the treadmill). Third, at the neuronal population level, the magnitude and temporal profiles of the firing rate modulations were similar during hand-guided and well-trained conditions. This suggests that the striatal units recorded received similar sensorimotor stimulation in both conditions. Fourth, in the training sessions that immediately followed the hand-guided sessions, correlations between firing rate and speed and position remained low even if the guiding plate was not used. Finally, it is not obvious how, in well-trained animals, low-level sensory input can account for linear changes in striatal firing rate versus position and speed. For instance, in the three illustrative speed-correlated units shown in Figure 4, spiking activity was not rhythmical and therefore cannot be explained by the locomotion-related dynamics of limbs, head or whiskers ${ }^{35,36}$.

It could also be argued that the position and/or speed correlates are related to reward expectation and that the lack of rewards in the handguided sessions is responsible for the weak position and speed representations. Such a possibility is not supported by the fact that, at the population level, firing rates were similarly modulated in well-trained and hand-guided animals throughout the entire execution of the task. In addition, in the recordings performed during learning, many trials were rewarded, but position and speed correlates remained weak for several sessions before appearing. Conversely, the fact that speed and position representations did not drop during the penalty part of error trials in well-trained animals (Supplementary Fig. 7) is incompatible with a major contribution of reward expectation. Finally, in our task, the linear correlations between firing rate and speed or position could not be mistaken for reward expectation signals. If this was the case, the speed-sensitive cells would not decrease their firing rate at the very end of the task when animals slowed down (Fig. $4 a-c$ and Supplementary Fig. 7). Given that rats occupied the front of the treadmill at the beginning and end of the trials, two times at which reward expectation is opposite, position representation can be isolated from reward expectation. In conclusion, our data are not compatible with the possibility that speed- and position-related activities in the DLS of well-trained animals simply reflect the stereotypical structure of movements or reward expectations. They are integrative signals acquired following extensive practice of the running sequence.

What could be the function of these signals? The linear correlations between firing rate and position are likely to provide contextual signals that inform the rat of its position relative to the front and the rear portions of the treadmill, which are important spatial landmarks for successful performance ${ }^{37-39}$. The integration of such signals with a movement-related spiking activity could constrain or modulate the performance of the animal: if running speed is too fast or too slow in a certain portion of the treadmill, the integration of the speed- and position-related striatal activity in downstream brain regions would modulate motor commands either at cortical or subcortical levels. This hypothesis is supported by the fact that the speed-related activity in the DLS surrounds changes in running speed. Moreover, DLS perturbation with muscimol increased trial-by-trial variability of running trajectories and altered the animals' capacity to modulate their performance after error trials, but spared the average front-rear-front structure of the sequence. The constraining/modulatory function of the DLS that we propose, along with its underlying neuronal mechanism, extends to motor habits a recent model of the role of the basal ganglia in motor learning ${ }^{40}$. In this model, which is mainly derived from experiments in the songbird, the striatum combines efferent motor copies and context signals to reinforce and modulate motor plans outside of the basal ganglia ${ }^{40}$. We hypothesize that, during extensive practice of a learned motor sequence, coincident efferent motor copies and contextual signals in the DLS cause high-level integrative representations of the most important aspects of the learned motor sequence: in the case of our task, the animal's running speed and position on the treadmill and, to a lesser extent, time.

A constraining/modulatory function of contextual and movement-related representations in the DLS, distinct from action generation or action selection, is consistent with the observation that Parkinson's disease patients perform reaching movements with preserved spatial accuracy, but present a specific deficit in speedselection mechanisms ${ }^{41}$ and with studies in rodents and non-human primates suggesting a specific contribution of the basal ganglia to the vigor of movements ${ }^{12,42}$. On the other hand, our results contrast with prominent works in rodents that suggest that the dorsal striatum only encodes the beginning and ending of action sequences through movement-independent signals that control actions, similar to the way traffic lights control road traffic ${ }^{4,6,7}$. One possible explanation for such discrepancy is that the tasks used ( $T$ maze and sequence of lever presses) required limited motor control in the middle portion of the sequence. In support of this explanation, when mice were trained to perform faster lever-press sequences, modulation of striatal activity appeared in the middle of the sequence ${ }^{43}$. Our observation that spiking activity in the DLS is continuously modulated by kinematic and contextual parameters during sequence execution might also seems at odds with studies reporting dominant striatal activity that preceded action initiation or cancellation ${ }^{44,45}$. Here we provide evidence that continuous task-related signals modulate motor execution, and our work is therefore compatible with a striatal race or accumulative model of action control ${ }^{46}$ that, in the case of tasks requiring brief movements ${ }^{44,45}$, would occur before action initiation. We further developed this concept by showing that, after extended practice of a motor sequence, the DLS can guide movement execution through continuous integration of multiple task-relevant information. Although the role of the DLS in habit formation has been known for a long time, our results suggest a straightforward neuronal mechanism to underlie such function and provide a new framework for understanding the role of the basal ganglia in motor control.

\section{METHODS}

Methods and any associated references are available in the online version of the paper.

Note: Any Supplementary Information and Source Data files are available in the online version of the paper.

\section{ACKNOWLEDGMENTS}

We thank J. Krakauer, K. Diba, J. Epsztein, O. Manzoni and H. Martin for excellent discussions and critical reading of this and/or earlier version of the manuscript, 
I. Cordon Morillas, C. Sales-Carbonell and L. Lalla for help with training of the animals, J. Perez-Ortega for help with LabView Vision, A. Brovelli for advice with multiple regression analysis, and R. Martinez for technical help. This work was supported by the Spanish Ministerio de Ciencia e Innovación (Ramon-Y-Cajal program, D.R.) EU-fp7 (International Reintegration Grant, IRG230976, D.R.; Marie Curie International Incoming Fellowship, IIF253873, P.E.R.-O.), INSERM (Avenir Program, D.R.), European Research Council (ERC-2013-CoG - 615699_NeuroKinematics, D.R.) and the Mexican Consejo Nacional de Ciencia y Tecnología (P.E.R.-O.).

\section{AUTHOR CONTRIBUTIONS}

P.E.R.-O. performed the experiments. D.R. and P.E.R.-O. designed the experiments, analyzed the data and wrote the manuscript.

\section{COMPETING FINANCIAL INTERESTS}

The authors declare no competing financial interests.

Reprints and permissions information is available online at http://www.nature.com/ reprints/index.html.

1. Woodworth, R.S. The Accuracy of Voluntary Movement. Psychol. Rev. III, 1-120 (1899).

2. Lee, T.D. Motor Control in Everyday Actions (Human Kinetics, 2011).

3. Bailey, K.R. \& Mair, R.G. The role of striatum in initiation and execution of learned action sequences in rats. J. Neurosci. 26, 1016-1025 (2006).

4. Barnes, T.D., Kubota, Y., Hu, D., Jin, D.Z. \& Graybiel, A.M. Activity of striatal neurons reflects dynamic encoding and recoding of procedural memories. Nature 437, 1158-1161 (2005)

5. Costa, R.M., Cohen, D. \& Nicolelis, M.A.L. Differential corticostriatal plasticity during fast and slow motor skill learning in mice. Curr. Biol. 14, 1124-1134 (2004).

6. Jin, X. \& Costa, R.M. Start/stop signals emerge in nigrostriatal circuits during sequence learning. Nature 466, 457-462 (2010).

7. Jog, M.S., Kubota, Y., Connolly, C.I., Hillegaart, V. \& Graybiel, A.M. Building neural representations of habits. Science 286, 1745-1749 (1999).

8. Packard, M.G. \& McGaugh, J.L. Inactivation of hippocampus or caudate nucleus with lidocaine differentially affects expression of place and response learning. Neurobiol. Learn. Mem. 65, 65-72 (1996).

9. Yin, H.H. et al. Dynamic reorganization of striatal circuits during the acquisition and consolidation of a skill. Nat. Neurosci. 12, 333-341 (2009).

10. Tang, C., Pawlak, A.P., Prokopenko, V. \& West, M.O. Changes in activity of the striatum during formation of a motor habit. Eur. J. Neurosci. 25, 1212-1227 (2007).

11. Deffains, M., Legallet, E. \& Apicella, P. Modulation of neuronal activity in the monkey putamen associated with changes in the habitual order of sequential movements. J. Neurophysiol. 104, 1355-1369 (2010).

12. Desmurget, M. \& Turner, R.S. Motor sequences and the basal ganglia: kinematics, not habits. J. Neurosci. 30, 7685-7690 (2010).

13. Miyachi, S., Hikosaka, O., Miyashita, K., Kárádi, Z. \& Rand, M.K. Differential roles of monkey striatum in learning of sequential hand movement. Exp. Brain Res. 115, 1-5 (1997).

14. Miyachi, S., Hikosaka, O. \& Lu, X. Differential activation of monkey striatal neurons in the early and late stages of procedural learning. Exp. Brain Res. 146, 122-126 (2002).

15. Lehéricy, S. et al. Distinct basal ganglia territories are engaged in early and advanced motor sequence learning. Proc. Natl. Acad. Sci. USA 102, 12566-12571 (2005).

16. Wymbs, N.F., Bassett, D.S., Mucha, P.J., Porter, M.A. \& Grafton, S.T. Differential recruitment of the sensorimotor putamen and frontoparietal cortex during motor chunking in humans. Neuron 74, 936-946 (2012).

17. Graybiel, A.M. Habits, rituals, and the evaluative brain. Annu. Rev. Neurosci. 31, 359-387 (2008)

18. Shmuelof, L. \& Krakauer, J.W. Are we ready for a natural history of motor learning? Neuron 72, 469-476 (2011).

19. Turner, R.S. \& Desmurget, M. Basal ganglia contributions to motor control: a vigorous tutor. Curr. Opin. Neurobiol. 20, 704-716 (2010).

20. Gerfen, C.R. \& Bolam, J.P. The neuroanatomical organization of the basal ganglia. in Handbook of Basal Ganglia Structure and Function (eds. Steiner, H. \& Tseng, K.) 3-28 (Academic Press, 2010).
21. Winn, P., Wilson, D.I.G. \& Redgrave, P. Subcortical connections of the basal ganglia. in Handbook of Basal Ganglia Structure and Function (eds. Steiner, H. \& Tseng, K.) 397-408 (Academic Press, 2010).

22. Shadmehr, R. \& Krakauer, J.W. A computational neuroanatomy for motor control. Exp. Brain Res. 185, 359-381 (2008).

23. Yin, H.H. \& Knowlton, B.J. The role of the basal ganglia in habit formation. Nat. Rev. Neurosci. 7, 464-476 (2006).

24. Balleine, B.W. \& Dickinson, A. Goal-directed instrumental action: contingency and incentive learning and their cortical substrates. Neuropharmacology 37, 407-419 (1998).

25. Apicella, P., Scarnati, E., Ljungberg, T. \& Schultz, W. Neuronal activity in monkey striatum related to the expectation of predictable environmental events. J. Neurophysiol. 68, 945-960 (1992).

26. Apicella, P., Ljungberg, T., Scarnati, E. \& Schultz, W. Responses to reward in monkey dorsal and ventral striatum. Exp. Brain Res. 85, 491-500 (1991).

27. West, M.O. et al. A region in the dorsolateral striatum of the rat exhibiting singleunit correlations with specific locomotor limb movements. J. Neurophysiol. 64, 1233-1246 (1990).

28. Shi, L.H., Luo, F., Woodward, D.J. \& Chang, J.Y. Neural responses in multiple basal ganglia regions during spontaneous and treadmill locomotion tasks in rats. Exp. Brain Res. 157, 303-314 (2004).

29. Whitlock, J.R., Pfuhl, G., Dagslott, N., Moser, M.-B. \& Moser, E.I. Functional split between parietal and entorhinal cortices in the rat. Neuron 73, 789-802 (2012).

30. Cho, J. \& West, M.O. Distributions of single neurons related to body parts in the lateral striatum of the rat. Brain Res. 756, 241-246 (1997).

31. Gage, G.J., Stoetzner, C.R., Wiltschko, A.B. \& Berke, J.D. Selective activation of striatal fast-spiking interneurons during choice execution. Neuron 67, 466-479 (2010).

32. Freeze, B.S., Kravitz, V., Hammack, N., Berke, J.D. \& Kreitzer, C. Control of Basal Ganglia Output by Direct and Indirect Pathway Projection Neurons. J. Neurosci. 33, 18531-18539 (2013).

33. Kravitz, A.V. et al. Regulation of parkinsonian motor behaviours by optogenetic control of basal ganglia circuitry. Nature 466, 622-626 (2010).

34. McGeorge, A.J. \& Faull, R.L.M. The organization of the projection from the cerebral cortex to the striatum in the rat. Neuroscience 29, 503-537 (1989).

35. Ranade, S., Hangya, B. \& Kepecs, A. Multiple modes of phase locking between sniffing and whisking during active exploration. J. Neurosci. 33, 8250-8256 (2013).

36. Ledberg, A. \& Robbe, D. Locomotion-related oscillatory body movements at 6-12 Hz modulate the hippocampal theta rhythm. PLoS ONE 6, e27575 (2011).

37. Berke, J.D., Breck, J.T. \& Eichenbaum, H. Striatal versus hippocampal representations during win-stay maze performance. J. Neurophysiol. 101, 1575-1587 (2009).

38. van der Meer, M.A., Johnson, A., Schmitzer-Torbert, N.C. \& Redish, A.D. Report triple dissociation of information processing in dorsal striatum, ventral striatum, and hippocampus on a learned spatial decision task. Neuron 67, 25-32 (2010).

39. Yamin, H.G., Stern, E.A. \& Cohen, D. Parallel processing of environmental recognition and locomotion in the mouse striatum. J. Neurosci. 33, 473-484 (2013).

40. Fee, M.S. Oculomotor learning revisited: a model of reinforcement learning in the basal ganglia incorporating an efference copy of motor actions. Front. Neural Circuits 6, 38 (2012)

41. Mazzoni, P., Hristova, A. \& Krakauer, J.W. Why don't we move faster? Parkinson's disease, movement vigor, and implicit motivation. J. Neurosci. 27, 7105-7116 (2007).

42. Wang, A.Y., Miura, K. \& Uchida, N. The dorsomedial striatum encodes net expected return, critical for energizing performance vigor. Nat. Neurosci. 16, 639-647 (2013).

43. Jin, X., Tecuapetla, F. \& Costa, R.M. Basal ganglia subcircuits distinctively encode the parsing and concatenation of action sequences. Nat. Neurosci. 17, 423-430 (2014).

44. Schmidt, R., Leventhal, D.K., Mallet, N., Chen, F. \& Berke, J.D. Canceling actions involves a race between basal ganglia pathways. Nat. Neurosci. 16, 1118-1124 (2013).

45. Cui, G. et al. Concurrent activation of striatal direct and indirect pathways during action initiation. Nature 494, 238-242 (2013).

46. Ding, L. \& Gold, J.I. The basal ganglia's contributions to perceptual decision making. Neuron 79, 640-649 (2013). 


\section{ONLINE METHODS}

All experimental procedures were conducted in accordance with standard ethical guidelines (European Communities Directive 86/60-EEC) and were approved by ethical committees (Ministère de l'enseignement supérieur et de la recherche, France, Ref 00172.01; Comité d'Experimentació Animal, Universitat de Barcelona, Spain, Ref 520/08).

Behavioral apparatus. The treadmill (Dattica) was $80 \mathrm{~cm}$ long and $14 \mathrm{~cm}$ wide and was surrounded by plexiglas walls $50 \mathrm{~cm}$ high. There was no resting platform and the only walkable area was the treadmill belt, which was driven by a brushless digital motor (BG 44 SI, Dunkermotoren) controlled by a custom-made program (LabVIEW, National Instruments) and a multifunction computer board (PXI6254, National Instruments). A 60-W light bulb illuminated the whole apparatus. One wall of the treadmill was equipped with a liquid well to deliver drops of sucrose solution (Fig. 1a). A photodetector positioned at $10 \mathrm{~cm}$ from the front wall signaled the first entrance of the animal in the so-called stop area in each trial (Fig. 1a). A warning sound (1.5 kHz, $65 \mathrm{~dB})$ signaled incorrect early entrances in the stop area.

Behavioral training. Animals were handled ( $2 \mathrm{~h} \mathrm{~d}^{-1}$ for $5 \mathrm{~d}$ ), familiarized to run on the treadmill at increasing speeds and trained to perform the task. During training, the treadmill speed was fixed for each animal $\left(35-40 \mathrm{~cm} \mathrm{~s}^{-1}, 40-160\right.$ trials per session, 1-2 sessions per day). Trials started independently of the animal's position, but, after a few training sessions, rats spent most of the inter-trial periods in the stop area. We established a criterion of performance accuracy $(\geq 72.5 \%$ of correct trials over the last 40 trials, for $\geq 3$ consecutive sessions). During training, the experimenter was not physically present in the behavioral room.

Animals. Long-Evans rats ( $n=15$, male, $250-400 \mathrm{~g}$ ) were housed in pairs (individually after surgery) in stable conditions of temperature $\left(22^{\circ} \mathrm{C}\right)$ and humidity (60\%) with a constant light-dark cycle ( $12 \mathrm{~h}: 12 \mathrm{~h}$, all experimental procedures were performed during the light phase) and free access to food and water. Five rats were used for electrophysiological recordings: rats 1 and 4 completed at least 127 sessions before the start of electrophysiological recordings; rats 5, 15 and 17 were used in the hand-guided control task and were implanted immediately after the habituation period; after eight hand-guided sessions, rat 17 was also trained and recorded in the regular version of the task and reached the learning criterion after 41 sessions. Rats 7-10 were over-trained for at least 40 sessions after reaching the learning criterion and then chronically implanted with cannulae in the DLS. Rats 13 and 14 were naive at the time of cannulae implantation and were used to determine the effects of intra-DLS injection of muscimol on locomotion. Rats 2, $6,7,9,10,22$ and 24 were used to probe the habitual nature of behavior.

Probing the habitual nature of the learned behavior. Rats 2, 22, 24 were trained to learning criterion and performed at least 25 sessions of overtraining. Then the treadmill was shortened by moving the front wall $30 \mathrm{~cm}$ toward the rear portion of the treadmill. Rats 7, 9 and 10 were trained with goal times at $4 \mathrm{~s}(180$ sessions), $5 \mathrm{~s}$ (115 sessions) and $4 \mathrm{~s}$ (130 sessions), respectively. After training, goal time was changed to $7 \mathrm{~s}$. At a different time during training, rats 6, 7, 9, 10, 22 and 24 were given unlimited access to the sucrose solution $\sim 24 \mathrm{~h}$ prior to the reward devaluation session. During these sessions sucrose was not delivered at the end of the correct trials.

Implantation of tetrode arrays for unit recordings in behaving animals. Under deep isoflurane anesthesia, a $2 \times 4$ tetrode array (nichrome wires, $12.5-\mu \mathrm{m}$ diameter, California Fine Wire) loaded on a NLX-9 micro-drive (Neuralynx) was implanted above the dorsal striatum (Supplementary Fig. 3a) through a craniotomy centered at $\mathrm{ML}= \pm 3.6 \mathrm{~mm}$ and $\mathrm{AP}=+0.6 \mathrm{~mm}$ from bregma. Tetrode tips were gold plated to reduce their impedance to $100-200 \mathrm{k} \Omega$ at $1 \mathrm{kHz}^{47}$. Two miniature screws implanted above the cerebellum served as ground and reference. After recovery from the surgery (1-2 weeks), the tetrodes were lowered toward the DLS $\left(25-100 \mu \mathrm{m} \mathrm{d}^{-1}\right)$.

Data acquisition and processing. Wide-band $(0.1-8,000 \mathrm{~Hz})$ neurophysiological signals from the tetrode arrays were amplified 1,000 times via a Plexon VLSI headstage and a PBX2 amplifier and continuously acquired at $20 \mathrm{kHz}$ on two synchronized National Instruments A/D cards (PCI 6254, 16 bit resolution).
Spike sorting was performed semi-automatically ${ }^{48}$ using the clustering software KlustaKwik (http://klustakwik.sourceforge.net) and the graphical spike sorting application Klusters (http://neurosuite.sourceforge.net/) ${ }^{49}$.

Animal's position, running speed and acceleration. To determine the position of the animals, we used a CCD camera (scA640-70fc, Basler, 60 frame $\mathrm{s}^{-1}, 9$ pixels $\mathrm{cm}^{-1}$ ) positioned laterally to the treadmill and a fluorescent marker attached to the left forelimb. The marker's positions were extracted with a custom made program (Vision, National Instruments) and averaged in 400-ms-long sliding windows (the average duration of a step cycle). Animals' speed and acceleration were derived from the distance traveled every $250 \mathrm{~ms}$.

Firing rate modulation during task execution. Trials were aligned relative to treadmill onset and were divided into non-overlapping windows of $250 \mathrm{~ms}$. For each trial, the firing rate in each window (spike count / 0.25) was smoothed with a Gaussian kernel filter of s.d. $750 \mathrm{~ms}$ and instantaneous firing rates were averaged across trials. In a second approach, we computed perievent histograms with multiple points of references ${ }^{31}$. We restricted our analysis to trials in which the animals performed the archetypical front-rear-front sequence (typically $>70 \%$ of the trials). We automatically selected archetypical trials by using a template matching method based on the averaged front-rear-front sequence performed during the entire session. For each selected trial, we identified five consecutive landmarks: 1) light on, 2) treadmill on, 3) reach of the back of the treadmill, 4) acceleration to cross the treadmill, 5) reaching the stop area. Then, for each animal we calculated the average time interval between landmarks 2-3, 3-4, 4-5 across all selected trials. The average time intervals between consecutive landmarks were divided in 250-ms-long windows, determining for each animal, a fixed number of windows between landmarks. Next, all the selected trials were divided according to their respective landmarks times and the number of windows between landmarks. Finally, for each unit the instantaneous firing rate was computed, smoothed, transformed into Z-scores and averaged across trials.

Moment-to-moment correlation between firing rate and main task variables. Correlation plots shown in Figure 4 (middle panels) were computed from all the trials, binned in 250-ms-long windows, from treadmill onset to treadmill offset. In some cases (Figs. 3 and 6 and Supplementary Fig. 12), we restricted our analysis to trials or portions of trials displaying the archetypical front-rear-front sequence. To avoid biasing our correlation analysis with a minority of data points at the extreme range of the behavioral variables (for example, lowest and highest speeds), we calculated the distributions of speeds and positions during each session and restricted the analysis to windows for which the value of the variable of interest (speed and position) fell inside the 90th percentile of its distribution. Using the entire speed and position data did not affect the results. We used partial correlation analysis to quantify the specific correlation between firing rate and a single task-relevant variable (position, speed, acceleration or time), once the effects of the others variables have been removed. We found no significant differences when computing Pearson's and Spearman's correlation coefficients and we only reported Pearson's correlation coefficients. To quantify the extent to which the firing rate of the units was linearly correlated with a combination of task variables, we used multiple regression analysis. For each session, multicollinearity in the predictor variables (position, speed, acceleration or time) was discarded by calculating the variance inflation factor (median 1.1, range 1.0-1.63; accepted values $<5$ ), the detection-tolerance index (median 0.89, range 0.61-0.99; accepted values $>0.2$ ) and the conditional index of the Belsley collinearity test (median 2.03, range 1-5.67; accepted values < 10; Matlab codes from B. Lau, http://www.subcortex.net/research/code/collinearity-diagnostics-matlab-code). To test if striatal firing rates were generally influenced by more than one variable, we compared regression models that took into account a single predictor with models that took into account several (2,3 or 4$)$ predictors. This was done by computing the Akaike information criterion values for each model.

To test if changes in firing rate preceded running speed changes, for each trial, spike trains were systematically shifted by $100,250,500,1,000$ or $2,000 \mathrm{~ms}$, backward or forward relative to the behavioral data. Then partial correlation coefficients between running speed and firing rate were recalculated for each shift value. To quantify the timescale of the relationship between firing rate and running speed, spike times were jittered on a trial-by-trial basis and the jitter values were randomly chosen between $\pm 100,250,500,1,000$ or $2,000 \mathrm{~ms}$. 
The partial correlation coefficients between speed and firing rate were recomputed for the different jitter ranges. To compare partial and multiple correlation coefficients between hand-guided and well-trained animals, we restricted our correlation analysis to trials in which the animals performed the archetypical front-rear-front sequences.

All the results presented were reproduced when using different bin sizes $(50,100,250,500,750$ and $1,000 \mathrm{~ms}$, with a fixed smoothing window of $750 \mathrm{~ms})$ or different smoothing windows $(100,250,500,750$ and 1,000 ms, with a fixed bin size of $100 \mathrm{~ms})$.

Tuning curves of striatal units for position, speed and acceleration. For each recording session the ranges of position, speed and acceleration values were divided in 25 bins. The size of the bins was $3.6 \mathrm{~cm}$ for position, $5.8 \mathrm{~cm} \mathrm{~s}^{-1}$ for speed and $\sim 10 \mathrm{~cm} \mathrm{~s}^{-2}$ for acceleration. The first and last bins of acceleration were adjusted to exclude the $5 \%$ smallest and highest acceleration values. The total number of spikes fired in each bin was divided by the total time spent by the animal in that bin. The firing rate tuning curves obtained were smoothed with a Gaussian kernel filter whose s.d. was 4 bins. To test for the significance of these tuning curves, we used a shuffling/bootstrap procedure. For each trial, the spike train of the analyzed unit was circularized and the beginning of the trial was randomly chosen. Then, three surrogate tuning curves were recomputed (one for speed, one for position and one for acceleration). The operation was repeated 500 times to generate a global band of confidence (the $5 \%$ highest and lowest values of the $500 \times 3$ surrogates tuning curves). These procedures reduce multiple comparison issue ${ }^{50}$ and the possibility that the firing rate modulation by a given variable (for example, speed) is secondary to the modulation of firing rate by another variable (for example, position and acceleration). Next, we designed a method to directly test if the significant relationship between firing rate and a given behavioral variable of interest (for example, acceleration), can be accounted by the relationships of another behavioral variable (for example, speed or position) with both firing rate and the variable of interest. For instance, for the unit shown in Supplementary Figure 10a, the increase in firing rate for maximal deceleration values (right panel), could result from the combined increased firing rate when the animal is in the front portion of the treadmill (Supplementary Fig. 10a) and the fact that maximum decelerations tend to occur at the front of the treadmill when the animal finished the running sequence. First, we computed the probability distribution of acceleration conditioned on position. Then acceleration values were pseudo-randomly reassigned on the basis of the probability distribution of acceleration conditioned on the position values. By doing so, the relationship between acceleration and position was preserved but the fine relationship between firing rate and acceleration was altered. Once all the acceleration values were generated, a surrogate tuning curve of firing rate relative to acceleration was recomputed and plotted (thin blue dashed line in left panel of Supplementary Fig. 10g). This operation was repeated 100 times. We considered that if one of the 100 surrogate tuning curves displayed a stronger modulation than the original tuning curve the modulation was not significant. For the tuning curves for acceleration, the entire procedure was performed twice: once to address speed confound and once to address position confound. The same procedure was also performed for tuning curves for speed and position. In the case of the unit in Supplementary Figure 10h, we noticed that the nonlinear tuning curve for position (thick blue line, left panel) was largely explained by running speed (thin gray dashed lines, left panel). For all the tuning curves that remained significantly modulated, we systematically subtracted the mean surrogate tuning curve and examined if the correlation coefficient between tuning curve and the variable of interest improved.

Changes in striatal representations during learning. In rat 17 , to increase statistical power, units from 5 consecutive sessions were grouped together ( $25 \pm 5.8$ units per group), and we calculated the median value of their multiple correlation coefficients. For each session behavioral accuracy was estimated by computing the Pearson's correlation coefficients between all the possible pairs of trajectories performed in a single session and averaging all the correlation coefficients.

Reversible inactivation of the DLS in behaving animals. We bilaterally implanted guide-cannulae in the DLS (coordinates relative to Bregma: $\mathrm{AP}=0.4 \mathrm{~mm}$,
$\mathrm{ML}= \pm 4.0 \mathrm{~mm}, \mathrm{DV}=-3.5 \mathrm{~mm})$ of naive $(n=2)$ and well-trained animals ( $>4$ months of training, $n=4$ ) under deep isoflurane anesthesia. The injectors protruded the guide-cannulae by $1 \mathrm{~mm}$. Local injections $(1 \mu \mathrm{l}$ per site at $0.2 \mu \mathrm{min}^{-1}$ ) were performed $10 \mathrm{~min}$ before the behavioral sessions. In naive animals, the $\mathrm{GABA}_{\mathrm{A}}$ agonist muscimol (Tocris) was diluted in saline and injected at different concentrations $\left(50 \mathrm{ng} \mu \mathrm{l}^{-1}, 500 \mathrm{ng} \mu \mathrm{l}^{-1}, 1 \mu \mathrm{g} \mu \mathrm{l}^{-1}\right.$ ). The highest dose induced potent motor impairments (rigidity, catalepsy). The two lowest doses had no apparent effect on basic locomotor activity in the home cage. To further characterize the effects of the lowest doses, animals were video-recorded while running on the treadmill. During these sessions the treadmill was started every minute during $20 \mathrm{~s}$ at a fixed speed of $35 \mathrm{~cm} \mathrm{~s}^{-1}$. Two blocks of 30 trials were separated by $30 \mathrm{~min}$ of rest in the home cage. Before the first block of trials, animals received bilateral injections of saline and before the second block animals were injected with muscimol. To compare the motor behavior between saline and muscimol injections (Supplementary Fig. 13), we subtracted the distribution of speeds (or positions) obtained during the saline and muscimol blocks. We generated surrogate muscimol and saline distributions by randomly selecting 15 trials from each condition and subtracted the distributions obtained. We repeated this procedure 300 times and determined a global band of confidence (the $5 \%$ highest and lowest values of the surrogate differences).

To quantify the stereotypy of the speed time courses during a single session, Pearson's correlation coefficients were computed for all the possible pairs of trials (we restricted our correlation to the last $2.5 \mathrm{~s}$ before entrance time). The variance of these correlation coefficients was used as a measurement of running speed stereotypy. We quantified the animals' ability to adjust their behavior after error trials (arrival time < goal time, early trials) and after trials with abnormally late entrance times (arrival time $>$ goal time $+3 \mathrm{~s}$, late trials). We defined an adjustment index as the difference in entrance times between the early (or late) trials and the next trial, divided by the size of the mistake (goal time - early (or late) arrival time). Our analysis was based on 93 early and 87 late trials from 7 muscimol sessions and 224 early and 97 late trials from 30 control experiments. Data from control conditions (before muscimol, before and after saline injections) were pooled together due to the low number of early and late trials during these sessions.

Histology. Animals were deeply anesthetized and transcardially perfused with PBS followed by $4 \%$ paraformaldehyde ( $\mathrm{wt} / \mathrm{vol}$ ). Cresyl violet staining of coronal sections $(60 \mu \mathrm{m})$ was performed. To quantify muscimol's diffusion, we injected $1 \mu \mathrm{l}$ of a solution containing 1,1'-Dioctadecyl-3,3,3',3'-tetramethylindocarbocyanine perchlorate (DiI, $50 \mathrm{mM}$ ) in two animals.

General statements on data collection and statistics. No statistical methods were used to pre-determine sample sizes, but our sample sizes (number of animals and total number of recorded units) are similar to those reported in previous publications $s^{4,731,38,39}$. Data collection could not be performed blindly, but, in most of the cases, the experimenter was not present in the experimental room during data collection. Moreover, analysis of the spiking activity and behavior was performed offline and Matlab programs were run in batch mode on all the data, independently of experimental conditions. The statistical tests used do not assumed normality of data distributions (which were always shown). The behavioral and neuronal data along with the Matlab codes used to generate the figures are available upon request to the corresponding author.

A Supplementary Methods Checklist is available.

47. Ferguson, J.E., Boldt, C. \& Redish, A.D. Creating low-impedance tetrodes by electroplating with additives. Sens. Actuators A Phys. 156, 388-393 (2009).

48. Harris, K.D., Henze, D.A., Csicsvari, J., Hirase, H. \& Buzsáki, G. Accuracy of tetrode spike separation as determined by simultaneous intracellular and extracellular measurements. J. Neurophysiol. 84, 401-414 (2000).

49. Hazan, L., Zugaro, M. \& Buzsáki, G. Klusters, NeuroScope, NDManager: a free software suite for neurophysiological data processing and visualization. J. Neurosci. Methods 155, 207-216 (2006).

50. Amarasingham, A., Harrison, M.T., Hatsopoulos, N.G. \& Geman, S. Conditional modeling and the jitter method of spike resampling. J. Neurophysiol. 107, 517-531 (2012). 